\title{
SURFACE FLOW VELOCITY MEASUREMENTS FROM UAV-BASED VIDEOS
}

\author{
J. Brauneck*, T. Gattung, R. Jüpner \\ Hydraulic Engineering and Water Management, Civil Engineering, TUK Kaiserslautern, Germany - (jens.brauneck, thomas.gattung, \\ robert.juepner)@bauing.uni-kl.de
}

KEY WORDS: Flow Velocity Measurements, Levee Breach, Hydrodynamic Modelling, LSPIV

\begin{abstract}
:
Most measuring methods for determining the volumetric flow rate or surface flow velocity have in common that they cannot be safely used under extreme outflow conditions. Especially in catastrophic situations, it is of particular interest to determine the amount of water that flows into the hinterland as precisely as possible in order to improve hydrodynamic models. Faulty assumptions lead to misleading calculations and may result in preventable casualties. As technical improvements throughout the last decade facilitated the widespread utilization of unmanned aerial vehicles (UAV) or remotely piloted aircraft systems (RPAS), these systems are now capable to collect and transmit precise information from remote areas to task forces immediately. The usage of a UAV is possible with minimal preparation at almost every place and is suitable for improving the database for a quick assessment of the status during a catastrophic event.

In this work, the determination of surface flow velocity using unmanned aerial vehicles (UAV) and floating optical tracers is evaluated. It is also discussed, to what extent numerical methods are able to efficiently undistort and correct this data. Precision analysis of video data from field investigations was performed with $\mathrm{R}$ using three different approaches that calculated the true velocity of the floating objects. The results indicate similar degrees of precision for both advanced methods but calculating an ortho-corrected video is a timeconsuming process not suitable for nearly real-time applications.
\end{abstract}

\section{MANUSCRIPT}

Levee failures are still difficult to be forecasted, especially specific modelling parameters are difficult to estimate (Ponziani and Bachmann, 2016). Therefore, it is hardly possible to prepare precise simulations in forecast quality in advance. Once a levee failure takes place, there is a high demand for the best possible estimation of the inundation process. In addition, there is a demand for data in order to implement emergency management measures in the process, e.g. procedures to close a levee breach. Here simulation techniques are an important tool to foresee the results of an action and assist the decision-making process. Particularly hydraulic models are powerful tools, if essential data of the failing levee and its surroundings is available (Tyrna et al., 2018). In the hydrodynamic simulation as well as in the real process, the conditions in the immediate vicinity of the levee failure have the highest influence on the progressing flood. The opening of the breach, its temporal development and the surface roughness along the main flow paths are substantially influencing the flood extent in general and the development in time (Bornschein et al., 2018) and (Huthoff et al., 2015).

Although generally accepted as essential tools for flood risk management, early warning systems and rapid mapping of large flooded areas, most remote sensing procedures are less suitable for the monitoring purposes of highly dynamic but rather small levee failures. Surveying and monitoring of presumably fragile structures and damaged areas on levees as well as the recording of the current flow characteristics cannot be fully guaranteed by means of satellites, helicopters and terrestrial exploration as the time gap between ongoing events and the comprehensive, continuous collection of relevant data prevails (Leitao et al., 2015). As technical improvements throughout the last decade facilitated the widespread utilization of unmanned aerial vehicles (UAV), also called unmanned aerial system (UAS) or remotely piloted aircraft systems (RPAS) when including the control device, these aerial platforms are now capable to collect and transmit precise information from non-accessible areas to the task

\footnotetext{
Corresponding author
}

force very quickly.

The vehicle itself can be either a multicopter system, generally equipped with four or more rotors, or a fixed wing system. The full potential of UAV-borne data integration in the field of disaster risk management and the acquisition of georeferenced data for hydrodynamic modelling is still poorly understood as only few investigations integrate the disciplines of close-range remote sensing and hydraulic modelling (Smith et al., 2014).

The need for further development of risk assessment in flood protection areas became obvious by the events in the Elbe-HavelJunction and Elbe-Saale-Junction during the Elbe flood disaster in June 2013 in Germany (Jüpner et al., 2017). There, a levee failure near the village of Breitenhagen was recorded using a rotorcraft UAV, instructed on behalf of the State Service for Flood Protection and Water Management in Saxony-Anhalt, Germany. Using photogrammetric ranging methods, we were able to analyse the visual UAV-flight information and set up a hydrodynamic model of the breach flow, based on four different digital surface models with extrapolated breach widths from 9 to 40 meters (Brauneck et al., 2016). By means of these calculations, the flow rate through the breach has been determined. Flow velocity and discharge are the most important characteristic values in analysing levee failures, its consequences and proper flood defence measures in the hinterland during an ongoing levee break.

The analysis of the fracture and its temporal evolution exposed the high sensitivity of the model results, since uncertainties in determining the inflow volume per unit time affect the entire propagation process and caused the confidence levels to drop significantly over time. It became obvious, that in order to improve these hydrodynamic models, supplementary information on the flow velocity is crucial. Despite a number of technical challenges, the quantity of projects focussing airborne surface flow measurement has increased steadily in recent years (Tauro et al., 2016a), especially those related to large-scale particle image velocimetry (LSPIV). As a principle that is well known and widely used in a lot of laboratory environments (Muste et al., 2008), LSPIV can be transferred to field 
investigations using permanently installed cameras or mobile terrestrial devices (e.g. telescopic poles) and best results can be achieved when a high number of artificially inserted tracers (like wooden chips) can be applied to the water surface. While the use of terrestrial or ground-based cameras reduces the time of postprocessing, it limits the areas of interest (AOI). Alternatively, as the most recent results confirm the reliability of the method for technically improved UAVs (Tauro et al, 2016b) as well as unaltered devices (Datert et al., 2017), but processing time is still an issue for all approaches.

As our emphasis is on timely data collection and rapid postprocessing using cameras from unaltered off the shelf multicopter $\mathrm{UAV}$, we concluded that tracing single floating objects is the most promising strategy for the above-mentioned purpose. During a flood event, airdropping of these tracers is possible using additional UAV, similar to those used for rescue operations (Microdrones, 2018) and (Westpac Little Ripper, 2018) or from helicopters.

The major challenge using UAV-based video recordings for flow velocity measurements is the instability of the camera during flight. The limited position accuracy of GNSS sensors, wind influences, and the controller's flight movements result in minimal movements of the UAV. A multicopter, when no control signal is received, always maintains its position and, when a passive position change is detected, actively directs it to return to its original position (Konrad et al., 2017). The autopilot system changes the rotational speed of one or more engines to direct the UAV to a different position. This movement results in pitching about the transverse axis or rolling around the longitudinal axis of the UAV. The attached camera transmits this movement to the video. Usually, a 3-axis gimbal is mounted between the hull of the UAV and the camera to compensate for these movements, but this mounting is, depending on quality and technology, not able to counteract for all roll and pitch movements of the multicopter (Beard and McLain, 2012). Drift movement of the drone is thus not compensated, which is why in this case the image footprint of the video shifts slightly. Any movements in the video thus distort a precise derivation of the surface velocity of the floating tracers, so position correction using geometrical transformation remains a crucial processing step.

\section{UAV-BASED MEASUREMENT OF SURFACE FLOW VELOCITY}

\subsection{Materials \& Methods}

In a series of field tests, the optical determination of the flow velocity was implemented with two UAVs of different categories. In the first measurement campaign, two sections of a branch of the river Nahe, close to Bretzenheim (Germany) were examined. The channel design of the first section is a near-natural trapezoidal water profile. At this point of the branch, the channel has a cross sectional flow width of $8.20 \mathrm{~m}$ and had a maximum depth of $0.70 \mathrm{~m}$. The riverbed is characterized by coarse gravel. The second measurement section was consistently shallow with maximum depths of about $0.3 \mathrm{~m}$. The entire section is located in a left turn so that the current is close to the right bank. Also in this section, the riverbed consists of coarse gravel. Due to the shallow water depth, the riverbed structures are visible through the water surface. Overflowed and circulated rocks caused local vortices and turbulent discharge behaviour, which seems to justify the above-mentioned assumptions concerning high turbulence and mean flow velocity.

For the determination of the surface flow velocity 26 inserted floats were analysed in 3 videos. After initial runs with table tennis and tennis balls, wooden chips with diameters from 5 to 8 $\mathrm{cm}$ and thicknesses of about $0.3 \mathrm{~cm}$ turned out as the most reasonable solution. The video from the first section was recorded using a DJI Phantom 3 Professional and includes experimental runs with 9 tracers. The Phantom 3 Pro is an off the shelf system for the private and hobby sector. Videos are recorded in $4 \mathrm{k}$ resolution at a maximum of 30 frames per second (fps) the camera is attached to a three-axis gimbal. In the second section, two videos with 11 and 8 tracers with a DJI Matrice 600 were recorded. In contrast to the former UAV, this hexacopter system is designed for the professional market due to the high payload of up to $6 \mathrm{~kg}$. A DJI Zenmuse $\mathrm{x} 5$ camera system, also equipped with a three-axis gimbal, was mounted underneath the multicopter. The second shot of the second section covered a larger section due to a higher flight altitude and spatial resolution. Thus, the measuring length could be elongated up to $35 \mathrm{~m}$.

During a flood event in January 2018 in Germany an additional measurement at the Bretzenheim test side concentrated on surface flow measurements before and after a culvert (section 3 ). Focusing on boundary conditions of a real flood event, measurements were implemented with minimal preparatory work. The only requirement for post-processing is geo-spatial information of at least two points in order to scale and locate the results in space. For this, the coordinates of ground control points at the bridge were derived by a georeferenced ortho-photo of the test side. To improve the identification of the floating tracer, coloured wooden chips with diameters from 0.05 to $0.08 \mathrm{~m}$ were used. Two videos were recorded, the first filming 15 wooden chips flowing into a culvert, which should be related to the flowing conditions entering a levee failure; the other one filming 15 chips leaving the culvert, which is used to be similar to turbulent conditions of water flowing out of a levee breach.

The analysis of the flow velocities was performed with the OpenSource Software Tracker (Tracker 4.11.0 GNU General Public License). Tracker is a free tool for video analysis based on the Open Source Physics (OSP) Java Framework. It compares userdefined image-patterns between frames and outputs corresponding parameters such as vectors, image coordinates and velocities (Brown and Cox, 2009) and (Claessens, 2017)].

The video data of section 1 and 2 was analysed using three different approaches that calculated the true velocity of the floating objects, where two of them eliminated the motion of the camera (figure 1). Common to all three approaches is a reduction to 5 frames per second (fps) since Tracker was not able to run at the high video resolution of $3,840 \times 2,160$ pixels and $30 \mathrm{fps} .5 \mathrm{fps}$ appeared to be the best compromise between excitability of the tracker software and the traceability of the floating tracers.

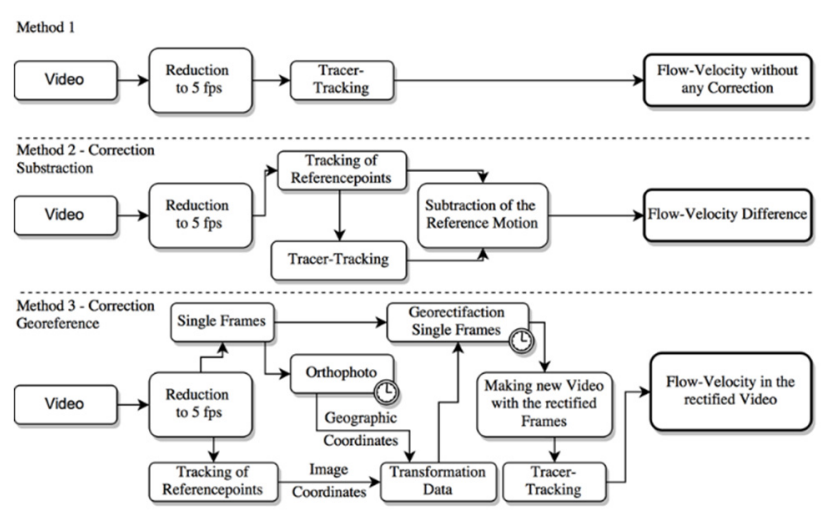

Figure 1 Workflows of three surface velocity determination approaches

Figure 1 shows the workflows of the following three velocity determination techniques used: 
- Approach 1) measured flow velocity at the surface without correction,

- Approach 2) derived surface flow velocity after simple correction via differential viewing of the recorded flow velocity with the recorded drone motion and

- Approach 3) derived surface flow velocity after elaborate drone motion correction via georectification of the video

Without correction, the speed of the floating particles in the water can be derived directly from the software Tracker (approach 1). Scaling can be performed using a calibration object or ground control points. With the scaling Tracker is able to transform the image coordinates into a metric coordinate system and by specifying the number of frames, the velocities are given directly in $\mathrm{m} / \mathrm{s}$. The auto tracking function of tracker enables the automatic derivation of velocities and acceleration by marking the objects to be tracked in individual frames.

Although assuming a rather static position of the UAV, the derived flow velocity is still subject to compensatory UAV movements. In order to determine the real flow velocity, two methodological approaches were examined. Firstly, a simple correction that subtracts the multicopter movement from the measured speed at each measurement point (approach 2). The calculation of UAV movement is based on the tracked movements of at least one reference point. This method is quick and easy to use but has the disadvantage that not all movements of the drone can be compensated. If the multicopter yaws heavily or if sudden changes in altitude occur, the consequential measurement errors are still present within the video after the correction.

A more complex georeferencing approach (approach 3) additionally corrects any further movements. The supposed advantage of this approach is that both UAV camera movements as well as optical distortion of the central perspective shot are eliminated. For the rectification of the video, an orthoimage of the test section is used as a reference that was created using the photogrammetric software "Agisoft PhotoScan Professional" (version 1.3.4). Agisoft PhotoScan, which is now renamed to Agisoft Metashape, uses the structure from motion photogrammetric method to reconstruct the position of the images based on several thousand points of control (Westoby et al., 2012), from which a digital surface model and the required orthophoto of the scene can be derived. This data is considered equal to the information that would be provided by emergency management authorities in Germany during a catastrophic event. In order to perform a geocorrection of the video into an orthorectified video (approach 3), a sufficient number of fixed points in the video must be mapped and reassigned with image coordinates. For this purpose, distinctive points are tagged and then tracked. Image tracking was performed using the Tracker software. Tracker is able to save the image coordinates of the tracked object per frame as a text file. In order to allow the best possible correction of the video, the points should be distributed equally over the entire video frame. The selected points were assigned to national coordinates using the orthophoto created in PhotoScan. The ArcGIS Model Builder requires text files to connect data to a national coordinate system. The text files are created in an automated R script (R 3.4.2 GNU General Public License). The program ArcMap (ESRI) is not able to correct entire videos. The movies must first be dissected into individual frames, which then can be geocoded. Iterating the model enables for an automation of this process so that each frame of the video is geocorrelated. In a final step, the frames are reassembled into a video with the frame count of $5 \mathrm{fps}$.

With the aim of comparing different processing approaches, distances travelled between uncorrected and geocorrelated video frames must be on the same scale. For this purpose, a local coordinate system is introduced in all videos. As an x-coordinate axis, an axis on the right bank was drawn through the designed ground control points (GCP). The y-axis is accordingly transverse to the flow direction.

In Tracker itself, it is possible to define the distance between two points as a reference scale. In the first section, the distance between the ground control points was exactly $30 \mathrm{~m}$, for the second section $20.57 \mathrm{~m}$. If the tracked data of the floating particles are exported as a text file, the image coordinates, the coordinates in the local system as well as the velocity are included. For evaluation purposes, the same floats were tracked in both videos and evaluated in $\mathrm{R}$.

The measured velocities were then compared without further correction with the velocities resulting from the use of the two correction approaches. In order to eliminate noise, a polynomial smoothing according to Savitzky and Golay was executed (Savitzky and Golay, 1964). This filter derives a kth degree polynomial over a series of $\mathrm{k}+1$ signals. Compared to other simple smoothing filters, high frequencies are not cut off but are moreover included in the calculation. As a result, this filter obtains the most important properties of the curve without flattening or shifting it.

The Nash-Sutcliffe Coefficient (NSC) (Equation 1) was used for a comparison of the velocity profiles (Nash and Sutcliffe, 1970). This coefficient is used primarily in hydrological modelling to compare the relationships between observed measurements and simulated data. It indicates to what extent the modelled runoff differs from the real runoff. In this case, it is also suitable to compare the relationship between the different recording methods and the velocity profiles. The Nash-Sutcliffe coefficient generates values between $-\infty$ and 1 .

$$
E=1-\frac{\sum_{\mathrm{t}=1}^{\mathrm{T}}\left(\mathrm{V}_{\text {ortho, }}-\mathrm{V}_{\text {altit }}\right)^{2}}{\sum_{\mathrm{t}=1}^{\mathrm{T}}\left(\mathrm{V}_{\text {ortho, }}-\overline{\mathrm{V}}_{\text {ortho, }}\right)^{2}}
$$

where $\quad \mathrm{E} 1=\mathrm{V}_{\text {ortho }}$ is modeled velocity (approach 2 ), $\mathrm{V}_{\text {alt }}$ is uncorrected velocity (approach 1 )

E $2=V_{\text {ortho }}$ is modeled velocity (approach 3 ), $V_{\text {alt }}$ is uncorrected velocity (approach 1)

E $3=V_{\text {ortho }}$ is modeled velocity (approach 3 ), $V_{\text {alt }}$ is modeled velocity (approach 2).

A value of one indicates a perfect match of two methods. A value of zero, on the other hand, implies that the model approximates the match as if the mean of the reference represents them. Negative Nash-Sutcliffe coefficients represent a poor match of both sequences and indicate that the mean of the reference would have given a stronger correlation. Furthermore, maximum and minimum measured velocities were compared and the maximum distance between the different methods was calculated.

The velocity results from the geocorrelated video are handled as a reference of the respectively recorded surface flow velocities since this is closest to the true speed with correction of the multicopter movement and the optical distortion of the central perspective. On the one hand, the measured flow velocity in the orthovideo is compared with the measured velocity of the uncorrected video and, on the other hand, the two measured velocities of the corrected methods (approach 2 to approach 3 ).

\subsection{Results of optical surface flow velocity measurements}

Table 1 shows some measurement statistics for the derived velocities of section 1 and 2 . Due to only decent wind effect during our measurements, differences in our results are hardly noticeable. The first section measurements showed mean surface flow velocities of $0.64 \mathrm{~ms}^{-1}$ (9 tracks recorded); the second one 
with a more turbulent flow revealed mean velocities around 1.8 to $1.9 \mathrm{~ms}^{-1}$ (19 tracks recorded). Our focus in the evaluation of results will be the comparison of the velocity derived from the three different methods in order to determine the feasibility of these approaches, rather than the accuracy of the measurement itself.

\begin{tabular}{cccc}
\hline Approach & 1 & 2 & 3 \\
\hline Mean & 0.64 & 0.64 & 0.64
\end{tabular}

Section 1

Standard Dev. $\quad 0.08 \quad 0.08 \quad 0.08$

\begin{tabular}{cccc} 
Max & 0.85 & 0.81 & 0.81 \\
Min & 0.42 & 0.46 & 0.49 \\
\hline Mean & 1.85 & 1.83 & 1.84 \\
Standard Dev. & 0.11 & 0.10 & 0.07
\end{tabular}

Section 2

\begin{tabular}{|c|c|c|}
\hline Max & 2.01 & 2.01 \\
\hline Min & 1.73 & 1.66 \\
\hline
\end{tabular}

Table 1 Statistics of surface velocity measurements using three different approaches $\left(\mathrm{ms}^{-1}\right)$

Both velocity correction methods presented similar results in most cases. They both differ from the velocity derived from the uncorrected video. In the first section with a uniform flow along the stream Nash-Sutcliffe coefficients were not able to verify this result (table 2).

\begin{tabular}{|ccccc|}
\hline & NSC & C.1 & C. $2^{2}$ & C.3 $3^{3}$ \\
\hline Section 1 & Mean & -1.80 & -0.71 & 0.41 \\
& Standard Dev. & 4.65 & 1.62 & 0.61 \\
& Max & 0.83 & 0.83 & 0.94 \\
& Min & -13.80 & -3.37 & -0.92 \\
\hline \multirow{2}{*}{ Section 2 } & Mean & 0.95 & 0.95 & 0.98 \\
& Standard Dev. & 0.03 & 0.03 & 0.02 \\
& Max & 0.99 & 0.99 & 0.99 \\
& Min & 0.90 & 0.88 & 0.93 \\
\hline
\end{tabular}

1 Comp. 1: Approach 1 vs. Approach 2,

2 Comp. 2: Approach 1 vs. Approach 3,

3 Comp. 3: Approach 2 vs. Approach 3.

Table 2 Nash-Sutcliffe Coefficients for all approaches using section $1 \& 2$ measurements

The unvarying velocities in combination with the remaining noise, i.e. the movements of the multicopter, which could not be eliminated by the smoothing filter, leads to a falsification in the NSC (Table 2). Thus, only visible inspection and the comparison of the minimum and maximum measured velocities, as well as the maximum gap between the flow paths could provide an assessment of results. The mean maximum difference between the velocity comparing the corrected methods with each other is $0.05 \mathrm{~ms}^{-1}$ with a standard deviation of $0.01 \mathrm{~ms}^{-1}$ ). With a mean maximum difference of $0.1 \mathrm{~ms}^{-1}$ with a standard deviation of 0.01 $\mathrm{ms}^{-1}$ between the ortho-corrected velocities with the uncorrected profiles both corrected methods reduce the deviation by half.
The second section with generally higher flow velocities states more about the different methods. NSCs are more expressive due to the significant changes in velocity profiles caused by the more turbulent and faster current. Therewith the mean NSCs comparing the corrected profile is 0.99 with a standard deviation of 0.02. The NSCs checking a corrected method against the uncorrected velocities are lower with a mean of 0.95 and a standard deviation of 0.03 . The results appear similar but there are small differences calculable. Also the mean maximum difference confirms this assumption. Comparing both correction methods with each other, the resulting difference is only $0.07 \mathrm{~ms}^{-}$ ${ }^{1}$. In contrast, the mean maximum difference between the orthocorrected velocity profiles and the profile from the raw video is $0.13 \mathrm{~ms}^{-1}$, which also means an improvement in surface flow velocity derivation.

\subsection{Results of Section 3}

According to the results of the first and second field measurements only the quick correction method 2 (subtraction, as described in chapter 2) is used for surface flow velocity measurements of section 3 as it is supposed to simulate a multicopter UAV mission during a flooding event. Figure 4 shows the results of the measurements as well as their spatial distribution. Each cell of the generated local raster with a cell size of $32 \times 32 \mathrm{~cm}$ represents a generalization of the surface flow velocity measured. In case of multiple measurements in one cell, the mean value was chosen. In addition, the number of measurements per cell is showed as well as the standard deviation for cells with multiple measurements

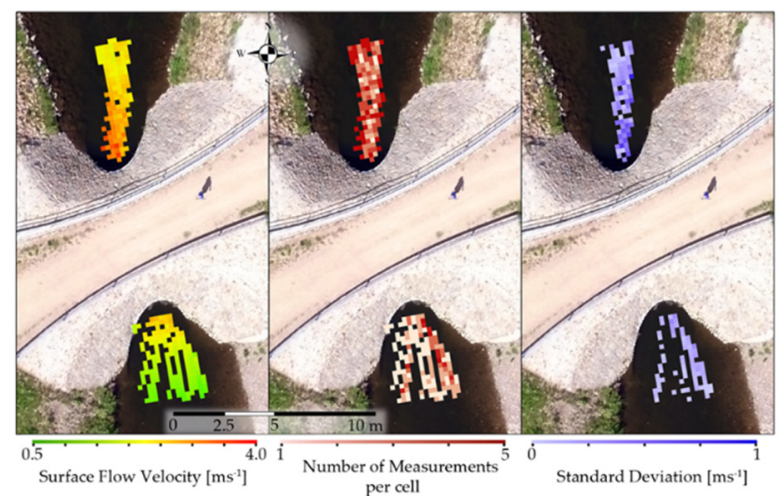

Figure 2 Results of the measurements of section 3 at the test side Bretzenheim. Flow direction is from south to north

The measured velocities are evenly distributed in front of the culvert. The water accelerates when flowing towards the culvert, starting with flow velocities of around 1 to $1.2 \mathrm{~ms}^{-1}$ and reaching a maximum speed of $2.9 \mathrm{~ms}^{-1}$. The maximum surface speeds of $3.7 \mathrm{~ms}^{-1}$ are measured right after the culvert. After the outlet the surface velocities indicate more turbulences that result in greater velocity differences in the immediate vicinity of the outlet. The further the water flows, the more it calms down again, with the result of more homogenous values and lower velocities of about $2 \mathrm{~ms}^{-1}$. According to the inhomogeneous surface velocity field directly after the culvert, the turbulences can also be distinguished looking at the plot of the standard deviation of the measurements. The variance of speed is higher all through turbulent flow conditions with swirls and vortexes that quickly modify the speed of the floating tracers. 


\subsection{Discussion}

All UAV-based measurements of flow velocities are generally error-prone caused by the movement of the flight platform. In this work we try to determine to what extent numerical methods are able to rectify and correct these data in a time-efficient manner. We do not determine the accuracy of the measurement itself. Any direct comparison between the different approaches is challenging due to the flow velocity differences along the flow profile, i.e. their spatial distribution, caused by unalike flow paths of the tracers. Because of field measurements, only in some areas of the second measurement section, where most of the tracers followed the main current, the different flow velocities of the tracers are comparable. The image-based tracking of the floating tracers with Tracker showed minimal inaccuracies, mostly because sometimes the centre of the tracer was not exactly detected, which led to "noise" within the speed flow. However, shallow water levels and a heterogeneous distribution of riffle sequences influence the flow behaviour even more so that along track flow velocities differ here as well. Therefore, the focus of the evaluation will be on the comparison of the differences of velocities derived with the three different methods, indicated by the NSC.

The results for the Nash-Sutcliffe Coefficients indicate similar finding for both sections, but due to the different flow velocities along the flow paths, the NSC values differ substantially. The flow paths as well as flow velocities in section 1 appear very homogeneous, with only minor variations along the track. As this is hydrologically considered a run section showing a smooth flow pattern without riffle or pool sections, the mean of flow velocity can be considered as a good measure for all tracks. As the NSC strongly compares against the mean of one approach, a highly significant mean will weaken the effects of the correction approaches. Therefore, the NSC of Comp. 1 and 2 show negative values. Although the velocity may differ at some areas, it remains steady along the flow path as it represents the typical flow profile of a river, with high flow velocities in the centre and decreasing velocities towards the sides. As these velocity profiles along the flow path show rather parallel patterns, significant improvement can only be achieved by eliminating the noise, which in this case would be the movement of the UAV. This reduction of movement leads to the increased NSC value of 0.41 for the comparison of approach 3 and 2 .

Section 2 shows generally higher velocity variabilities due to the presence of riffle sections and constantly varying velocities with a trend towards lower velocities at the end of the section. As all tracers follow the path of the main current line, similar velocities for all tracers at certain areas are the consequence. Minimum and maximum velocity values as well as standard deviation show a greater range at section 2. Due to this variability, the mean of the measurements does not reproduce the velocities and NSC values increase.

Although there is no major change in NSC statistics, the maximum differences in derived velocities indicate the impact of the correction approaches. In section 1 there are only minor improvements detectable, as stable flow conditions prevail throughout the flow path.

Section 2 shows declining differences for maximum velocities starting with maximum differences of $0.27 \mathrm{~ms}^{-1}$ between approach 1 and 2 towards maximum differences of $0.16 \mathrm{~ms}^{-1}$ when comparing approach 2 and 3 . The similar trend is visible comparing the minimum velocities and their differences. Between approach 2 and 3 the values show less than half of the difference compared to the results from approach $1 \mathrm{vs.} \mathrm{approach}$ 2. As minimum velocities increase and maximum velocities decrease, the data variability decreases with each correction approach. Due to the different hydrological nature of both sections, this effect is only visible in section 2, where higher surface velocities are present.

The most convenient weather conditions during our field campaign led to only small movements of the UAV. With increased winds, the effect of falsification due to UAV movement would be stronger and the correction should definitely be conducted in order to determine accurate surface flow velocities. Though, as our emphasis is on timely data collection and rapid post-processing using cameras from off-the-shelf UAV to support emergency management, we prefer approach 2 for a swift correction of the video data. Without correction, the derived surface flow velocity and the movement of the UAV can overlap, so that the results can differ considerably.

Figure 2 indicates mean velocities of about $1 \mathrm{~ms}^{-1}$ for the inundated areas in the vicinity of the levee failure in Breitenhagen, whereas velocities within the breach would be significantly higher. Due to the turbulent nature of levee failures and the occurring flow through the breach, the visibility of floating tracers within the breach is obviously questionable. The analysis of the video sequence of Breitenhagen however revealed relatively stable conditions before and after the breach, comparable to those in section 3. There, an acceleration of the floating tracers is visible throughout the underpass, which is in accordance with theoretical considerations.

With a focus on swift processing time, the simple correction method of subtraction of a fixed point from the tracked tracer provides the best result as it seems to be a good compromise between data quality and processing duration and can be calculated within minutes using any office PC compared to several hours for approach 3.

One major argument against the use of UAV during flooding events is a lack of weatherproof systems as most UAV are very vulnerable to moisture. Even though the Breitenhagen levee breach was recorded during pleasant weather conditions without any precipitation nearby, IP certified systems should be given preference when considering the purchase of an UAV for monitoring issues.

Similar considerations should be kept in mind for the improvement of this method for unfavourable lighting conditions. This could include adaptions in terms of fluorescent tracers or even using containers with supersaturated solution, e.g. heating pads, in combination with thermal infrared cameras.

\section{CONCLUSIONS}

After the evaluation of first field measurements, the use of floating objects to be tracked by the UAV can be considered as promising for levee breach flow analysis. The surface flow velocity during an event is still an unknown quantity with regard to the calculation of the breach discharge and the subsequent flooding of populated areas. Faulty assumptions lead to misleading calculations and may result in preventable casualties. As other methods of flow velocity measurement can only hardly be applied in the course of levee failures due to dangerous conditions, more effort should be made to verify and establish these airborne measurements as they could provide data for a wide range of models. This concept is not suitable for an in-depth analysis of surface flow patterns but to quickly derive reliable values for a specific incident with volunteer pilots from different backgrounds.

We demonstrated that UAV based flow velocity measurements can be achieved using off the shelf systems. The simplified flow velocity determination using solitary fixed reference points with tracer objects shows a clear potential as a rapid data acquisition method under challenging circumstances that could be used for levee breaches as for the purpose of near real time modelling approaches. Significant improvement considering processing 
time as well as accuracy is expected for the use of real time kinematic (RTK) positioning devices as onboard systems for UAV. The RTK positioning would lead to a more static recording and most likely immediate derivation of surface flow velocities. But since we focus on off-the-shelf UAV systems, these improvements need to be discussed elsewhere.

Future research on the topic of UAV based velocity measurements should include the setup of an integrating application, preferably for mobile devices, which speeds up the velocity determination by predefining the amount of reference points. An in depth analysis of the accuracies that are to be expected from these measurements compared to well-proven approaches is out top priority.

In terms of further developing real time modelling approaches, more research has to be done in the field of close range multi sensor applications. If the flight platform is equipped with supplementary multi- or hyperspectral recording systems, information about the surface conditions and the surface material could be obtained in addition to the spatial coordinates, which go beyond the purely visual interpretation. The complex and highly subjective process of model calibration could be shortened and hydro-numerical models would be much more objective and accurate. In order to support emergency management authorities, more details on the model accuracy must be determined by additional field measurements and sensitivity analyses.

\section{REFERENCES}

Beard, R., McLain, T.: Small unmanned aircraft: Theory and practice. Princeton Univ. Press, Princeton, NJ, 2012, +320 pp. doi: $10.1515 / 9781400840601$.

Bornschein, A., Pohl, R.: Land use influence on flood routing and retention from the viewpoint of hydrodynamics. J Flood Risk Management 2018, 11, 6-14. DOI: 10.1111/jfr3.12289.

Brauneck, J., Pohl, R., Juepner, R.: Experiences of using UAVs for monitoring levee breaches, IOP Conference Series: Earth and Environmental Science 2016, 46, Number 1. doi: 10.1088/17551315/46/1/012046.

Brown, D., Cox, A.: Innovative Uses of Video Analysis. The Physics Teacher, 2009, 47, 145-150. doi: 10.1119/1.3081296.

Claessens, T.: Analyzing Virtual Physics Simulations with Tracker. The Physics Teacher 2017, 55, 558-560. doi: 10.1119/1.5011834.

Detert, M., Johnson, E.D., Weitbrecht, V.: Proof-of-concept for low-cost and non-contact synoptic airborne river flow measurements. Int J Remote Sens 2017, 38, 8-10, 2780-2807. doi: $10.1080 / 01431161.2017 .1294782$.

Huthoff, F., Remo, J. and Pinter, N.: Hydrodynamic levee-breach and inundation modelling. J Flood Risk Management 2015, 8, 218. doi: $10.1111 / \mathrm{jfr} 3.12066$.

Jüpner, R.: Coping with extremes - experiences from event management during the recent Elbe flood disaster in 2013. In: Special issue "Land for Flood Risk Management - A catchmentwide and multi-level perspective", J Flood Risk Management 2017, eds. Hartmann, Schanze and Jilkova, Wiley, online, 7 pp. doi : 10.1111/jfr3.12286.

Konrad, T., Engelhardt, T., Abel, D.: Propeller thrust identification and calibration for high-precision control of a quadrotor unmanned aerial vehicle. 25th Mediterranean
Conference on Control and Automation MED, Valletta, Malta, 36 July 2017, 1225-1230. doi: 10.1109/MED.2017.7984285.

Leitao, J. P., Moy de Vitry, M., Scheidegger, A., Rieckermann, J.: Assessing the quality of Digital Elevation Models obtained from mini-Unmanned Aerial Vehicles for overland flow modelling in urban areas. Hydrol Earth Syst. Sci. Discuss. 2015, 12, 5629-5670. doi: 10.5194/hessd-12-5629-2015.

Microdrones - Test the waters with microdrones ${ }^{\circledR}$. Available online: https: //www.microdrones.com /en/landingpages/mdsar/ (accessed on 28.08.2018).

Muste, M., Fujita, I., Hauet, A.: Large-scale particle image velocimetry for measurements in riverine environments. Water Resour Res 2008, 44, W00D19. doi: 10.1029/2008WR006950.

Nash, J.E., Sutcliffe, J.V.: River Flow Forecasting Through Conceptual Models: Part 1. - A Discussion of Principles. $J$ Hydrol 1970, 10, 282-290. doi: 10.1016/0022-1694(70)90255-6.

Ponziani, M., Bachmann, D.: Real-time monitoring and forecasting of dike strength. J Saf Secur Eng 2016, 6, 122-131. doi: 10.2495/SAFE-V6-N2-122-131.

Savitzky, A., Golay, M.J.E.: Smoothing and Differentiation of Data by Simplified Least Squares Procedures. Analytical chemistry 1964, 36, 1627-1639. doi: 10.1021/ac60214a047.

Smith, M.W., Carrivick, J.L., Hooke, J., Kirkby, M.J.: Reconstructing flash flood magnitudes using 'Structure-fromMotion': A rapid assessment tool, J Hydrol 2014, 519, Part B, 1914-1927, ISSN 0022-1694. doi: 10.1016/ j.jhydrol.2014.09.078.

Tauro, F., Petroselli, A., Arcangeletti, E.: Assessment of dronebased surface flow observations. Hydrol Process. 2016, 30, 1114-1130. doi: 10.1002/hyp.10698.

Tauro, F., Porfiri, M., Grimaldi, S.: Surface flow measurements from drones. J Hydrol 2016, 540, 240-245, ISSN 0022-1694. doi: 10.1016/j.jhydrol.2016.06.012.

Tyrna, B., Assmann, A., Fritsch, K., Johann, G.: Large-scale high-resolution pluvial flood hazard mapping using the rasterbased hydrodynamic two-dimensional model FloodAreaHPC. $J$ Flood Risk Management 2018, 11: S1024-S1037. doi:10.1111/jfr3.12287.

Westoby, M., Brasington, J., Glasser, N., Hambrey, M., Reynolds, J.: 'Structure-from-Motion' photogrammetry: A lowcost, effective tool for geoscience applications. Geomorphology 2012, 179, 300-314. doi: 10.1016/j.geomorph.2012.08.021.

Westpac Little Ripper Lifesaver. Available online: https: //thelittleripper.com.au/ (accessed on 28.08.2018). 